\title{
Henry Bauchau et le Théâtre des Osses
}

Rendre compte des rapports entre l'écrivain Henry Bauchau et le Théâtre des Osses, sous l'impulsion de sa directrice Gisèle Sallin, c'est relater une rencontre. Il en va sans doute toujours ainsi dans l'histoire des interprétations ou des adaptations théâtrales: la vie des compagnies dramatiques se nourrit de passions partagées et l'histoire des représentations d'un texte sur scène est celle de l'investissement émotionnel, intellectuel et matériel de certains êtres qui ont le privilège, par chance mais aussi par vouloir, de rendre visibles et partageables leurs lectures. On commencera donc ici par évoquer, au départ des traces que livre la correspondance entre Gisèle Sallin et Henry Bauchau conservée au Fonds Henry Bauchau de l'UCL, mais aussi des interviews, des programmes et des différents paratextes disponibles, la rencontre entre deux créateurs. Il s'agit d'une histoire suisse, bien sûr, puisque Gisèle Sallin est Fribourgeoise et qu'elle a co-fondé le Théâtre des Osses en 1978 avec Véronique Mermoud, amenant le public suisse à découvrir l'œuvre d'Henry Bauchau et à s'enthousiasmer à son tour pour elle. Mais il s'agit, au-delà de cet ancrage particulier, de comprendre la dynamique qui sous-tend le type de travail théâtral entrepris et la particularité de ce qui fonde le rapport profond et durable entre la personnalité d'un metteur en scène et d'un écrivain.

\section{Le pouvoir de l'acte poétique et théâtral}

Au départ de cette rencontre, il y a un heureux hasard, ou ce qu'André Breton n'aurait pas manqué d'appeler un "hasard objectif». Car la place de l'œuvre de Bauchau dans l'histoire du Théâtre des Osses ne trouve pas son origine en Suisse mais en Belgique et au Québec, et non pas dans la production dramatique de Bauchau, mais dans son ouvre romanesque: en 1990, Giselle Sallin, qui faisait partie du jury d'un concours de jeunes auteurs belges, a reçu en cadeau, à la fin de sa mission, des livres d'auteurs belges, parmi lesquels Edipe sur la route, qu'elle a apporté dans l'avion qu'elle prenait ensuite pour Montréal. La lecture de ce livre a représenté pour elle le choc d'une révélation: elle ne l'a plus quitté. Revenue chez elle, elle a téléphoné au romancier pour lui dire qu'elle venait de parcourir «le plus beau livre de sa vie \$35. L'écrivain lui a alors révélé qu'un épisode de ce roman

356 Gisèle Sallin, Gisèle Sallin et Henry Bauchau. Histoire d'une rencontre artistique, juin 2009, 
avait été retiré pour en constituer un récit séparé, intitulé Diotime et les lions. Gisèle Sallin l'a lu aussitôt, et rappelé Henry Bauchau pour lui demander les droits de représentation avec Veronique Mermoud, l'une des figures marquantes du théâtre en Suisse, comme elle formée chez Benno Besson, et avec qui elle avait réalisé en 1986 un dossier analysant la situation théâtrale dans le canton de Fribourg, incluant la proposition de réaliser un centre dramatique professionnel régional.

Le 11 avril 1993, Gisèle Sallin annonce à Henry Bauchau, dans une longue lettre, que le Théâtre des Osses vient d'être créé à Givisiez pour une période de quinze ans. Dès les premiers mots, elle s'adresse à l'auteur pour "dire le bouleversement comme il vient. Faire savoir absolument l'importance de cette ouvre dans [sa] vie» $[\mathrm{B} 5272]^{357}$, et elle lui annonce son intention de monter Diotime:

D'abord ceci:

Je porterai Diotime à la scène. Véronique Mermoud interprétera le texte, tel qu'il est, sans adaptation, sans changer, sans omettre un seul mot. [B 5272]

On voit déjà ce qui restera la ligne de conduite du travail de Gisèle Sallin, et qui tient à un mot: le souci de la justesse. Dans cette lettre, dont il apparaît nécessaire de citer des passages importants, elle explique ce qui est au fondement de cette attitude, qui se comprend en regard de sa formation, et qui rejoint ce qui l'a profondément troublée à la lecture d'Edipe sur la route:

Je suis une passionnée de théâtre (de la vie aussi) et j'ai le bonheur de vivre de cette passion. Partie de rien, ma route a rencontré celle de Maria Casarès alors que je faisais mes premiers pas. Elle m'a transmis dans l'espace magnifique du théâtre des secrets: ... l'ÉVEIL.

L'éveil à la force... à la puissance du geste juste

L'éveil à la grâce du chant intérieur

(mes parents m'ont transmis le sens du rite et des mythes)

Un geste faux fait par Casarès nous montre l'ampleur du désastre et son désarroi est de l'ordre de l'effroi [...] elle cherche le geste juste, le juste chant c'est une question de survie. Quand elle trouve, cela s'inscrit dans l'espace pour toujours. C'est la trace humaine la plus bouleversante qui soit... C'est celle d'Alcyon, de Clios, d'CEdipe et des autres... [B5272 bis]

On comprend que ce qu'a perçu la lectrice de Bauchau, c'est sa faculté à raconter l'histoire d'êtres en quête de gestes et d'actions justes, c'est-à-dire accordés à leur nature profonde et à leur relation exacte à autrui, et qui sentent que le malheur qu'ils vivent et qu'ils provoquent autour d'eux provient de ce qu'ils n'atteignent pas cette adéquation à eux-mêmes et au monde. Or, elle observe que cette question, développée dans la fiction romanesque, n’a pu l'être que par un homme traversé

dossier de presse de Clios le bandit, Centre dramatique fribourgeois, Théâtre des Osses, 13 novembre-7 décembre 2008.

357 Nous livrons entre crochets les références des archives déposées au Fonds Henry Bauchau de l'UCL. 
par de telles affres dans sa propre vie, et qui a dû lutter pour construire, par son œuvre, le lieu de son authenticité:

Si votre ouvre a ce souffle, c'est qu'elle contient ce désir d'absolu d'être soimême et personne d'autre, désir auquel nous nous acharnons à renoncer et cela nous rend malades.

C'est pourquoi je dis qu'il y a là un pouvoir magique de guérison. Cette guérison s'opère grâce à l'irrésistible séduction.

Vous avez réussi ceci:

Etre soi-même, cette quête-là est une irrésistible séduction - c'est la plus grande, c'est ça VIVRE - et l'on atteint le jubilatoire... [B5274bis]

Elle exprime aussi, d'une manière générale, la nécessité qu'implique toute œuvre authentique de ne céder en rien sur son désir ${ }^{\mathbf{3 5 8}}$, pour reprendre les mots de Lacan qui désignait ainsi la hauteur d'âme d'Antigone, en même temps que la terrible exigence qui la situait dans l'ère du tragique.

Lorsqu'apparaît l'irrésistible séduction d'être soi-même au-delà de tout, c'est qu'il y a derrière tout cela une vérité. Cette vérité, c'est celle qu'accepte de vivre le créateur face à son œuvre. [B5274 ter]

Sans qu'il soit question ici de psychanalyse, on voit que Gisèle Sallin pointe la dimension auto-affective de l'œuvre d'Henry Bauchau, c'est-à-dire sa capacité à affecter le sujet parlant, qui se délie par le dire. Et ce qui se joue de «séduction" dans l'expression du désir d'être soi, c'est aussi sa performativité seconde, à savoir ce que Paul Ricour appelle “l'identité narrative»"359: la faculté qu'ont les hommes à construire le sens de leur vie grâce à la lecture de récits littéraires, qui leur fournissent "un modèle d'intelligibilité „360. C'est en ce sens que Gisèle Sallin comprend, d'une part, la dynamique de la parole en tant que thématique littéraire dans l'œuvre de Bauchau, où les protagonistes sont amenés à raconter leur propre histoire et ainsi à lui octroyer un sens, et d'autre part, les retombées performatives du récit romanesque sur le lecteur, appelé lui aussi à interroger et configurer sa propre existence en regard des références offertes dans la fiction:

[...] tous les personnages sont impliqués dans le déroulement des récits. Ils racontent leurs actes et le fait de les raconter les modifie. Lorsqu'CEdipe raconte le labyrinthe, il y retourne. Lorsque Diotime raconte son enfance et sa transformation en femme, elle revit tout. Ce que je pense depuis quelque temps, c'est que votre

358 Jacques Lacan, Le séminaire, livre VII : L'éthique de la psychanalyse, Paris, Le seuil, 1986, p.370. 359 "À la différence de l'identité abstraite du Même, l'identité narrative, constitutive de l'ipséité, peut inclure le changement, la mutabilité, dans la cohésion d'une vie. Le sujet apparaît alors constitué à la fois comme lecteur et comme scripteur de sa propre vie selon le vœu de Proust. Comme l'analyse littéraire de l'autobiographie le vérifie, l'histoire d'une vie ne cesse d'être refigurée par toutes les histoires véridiques ou fictives qu'un sujet se raconte sur lui-même. Cette refiguration fait de la vie elle-même un tissu d'histoires racontées. ", Paul Ricour, Temps et récit III, Le temps raconté, Paris, Le Seuil, 1985, pp. 442-443.

360 Paul Ricœur, Soi-même comme un autre, Paris, Le Seuil [1990], 1997, p. 192. 
œuvre a du pouvoir. Nous verrons bien, avec la réalisation de Diotime, si je dis vrai! [B5273]

En d'autres termes, ce qui attire Gisèle Sallin dans l'œuvre d'Henry Bauchau, c'est donc ce qu'elle contient potentiellement en tant qu'acte, en tant qu'impulsion vers l'authenticité et puissance de transformation, où elle perçoit sa théâtralité: "Ce qui me fascine dans l'écriture d'Henry Bauchau, c'est qu'elle est active", écrit-elle. "Au fur et à mesure du roman, elle modifie le lecteur. Dans ce sens, je trouve que c'est une écriture tout à fait théâtrale: elle modifie le personnage au fil du récit aussi bien que les spectateurs. „361

Cette particularité de l'œuvre la touche d'autant plus profondément qu'elle répond à ce qui lui apparaît être une lacune grave de la production culturelle dramatique de son époque. Le théâtre romand, sur lequel elle a été amenée à réfléchir longuement, lui semble en effet avoir perdu son dynamisme à force de mettre la nostalgie et la suspicion au cour de son propos. C'est contre cette asthénie du milieu dramatique qu'elle a voulu réagir en créant le Théâtre des Osses, et l'œuvre de Bauchau, tout entière investie d'une espérance dans le pouvoir actif du langage, à la fois thématisé et mis en jeu, lui semble rejoindre au plus intime ses attentes:

Il y a cinq ans, je me suis intuitivement mise en position de repli par rapport au théâtre romand car il y soufflait un vent de destruction incroyable. À Fribourg nous sommes une petite équipe et pendant que les autres se sont détruits, nous avons construit un projet, un lieu, parce que nous ne pouvons pas supporter que le théâtre meure. Or, il est en train de mourir, à cause du monde dans lequel on vit... parce que les gens de théâtre ont perdu les sens profonds des gestes, et des chants, et du jeu. Tous ont perdu le sens de l'ACTE. (En anglais, jouer = to act). [...] Il s'agit de remettre l'acte au centre de l'espace théâtral. Or l'ACTE doit être contenu dans l'écriture, dans le jeu, dans la mise en jeu.

Votre écriture, Henry Bauchau, est ACTE. [B5272 ter -B5273]

Ce qui autorise Gisèle Sallin à trouver dans l'écriture de Bauchau ce qui répond à son attente, c'est la qualité particulière de sa langue, qu'elle appelle «l'état de poésie", qui a disparu de nombreux textes de théâtre modernes qui ne témoignent plus d'aucune croyance dans la puissance du langage:

L'écriture théâtrale ne sait plus - sa forme s'est cristallisée. Nous nous sommes distanciés du désir collectif de poésie (la poésie étant bien sûr un acte) et nous n'avons plus les textes - c'est cela que m'a révélé la lecture d'Edipe sur la route. [...] Ce n'est pas vous qui manquez d'expériences théâtrales... c'est le théâtre qui a perdu l'état de poésie, qui a sclérosé ses formes pour que l'état de poésie ne pénètre plus... [B5274]

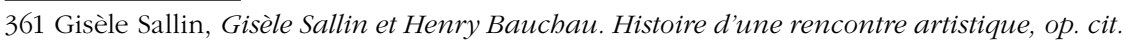


On comprend dès lors que ses choix de mises en scène se porteront au départ sur les textes inspirés d'Euripide (Médée, en 1982) ou de Sophocle (Antigone en 1988, Edipe roi en 1993). Mais il apparaît à Gisèle Sallin que le souffle poétique contemporain d'Henry Bauchau n'est pas moins prenant dans Edipe sur la route ou dans Diotime et les lions, lorsqu'il recourt à une langue d'une forte puissance incantatoire et évocatrice, et qu'il met en jeu le pouvoir guérisseur, ou simplement réconciliateur, de la parole et du chant qui osent dire les blessures en même temps que la quête de leur cicatrisation par le seul fait de les dire. Aussi, lorsqu'elle présente la création de Diotime et les lions, en, 1994, elle écrit dans le dossier de presse:

Le texte d'Henry Bauchau contient un savoir ancien qui a le pouvoir de guérir. Ce n'est pas ce texte qui doit être adapté au théâtre. C'est le théâtre qui doit s'adapter à lui. C'est le théâtre qui doit comprendre pourquoi, peu à peu, il a renoncé à vivre son rôle: celui de transmettre le désir de la réconciliation intime et publique ${ }^{362}$.

Dans sa correspondance, Gisèle Sallin exprime combien pour elle l'intime et le public sont en lien de manière indissoluble. Le 21 novembre 1996, dans un long fax qu'elle adresse à Henry Bauchau, elle décrit son travail en ces termes: "Je circule de l'infiniment privé à l'horriblement public et de l'infiniment public à l'horriblement privé» [B50402]. Ces mots touchent profondément Henry Bauchau qui répond le jour même: "J'ai trouvé très beau et passionné ce que vous m'écrivez du métier de metteur en scène. J'ai fait photocopier ce fax pour le garder et pouvoir le relire» [B5405]. C'est que de son côté, le romancier, qui tente une écriture d'après désastre et d'après psychanalyse, comprend de l'intérieur à quel point les failles intimes de l'être sont ce qui lui permet de devenir une figure publique nécessaire, et combien en retour, cette posture d'écrivain pèse sur la vie intime de l'homme, qu'elle dévore. La "paratopie „363 d'un écrivain qui se met à traduire ses rêves et ses hantises au grand jour, et la position du metteur en scène qui doit donner une forme extérieurement visible et partageable à un imaginaire d'écrivain verbalisé, s'avèrent sous l'angle particulier de l'articulation du public et de l'intime, dans une certaine similitude.

Chez Henry Bauchau, Gisèle Sallin retrouve quelque chose de la catharsis tragique antique qui tient, selon notre hypothèse, à la puissance empathique de son langage. L'œuvre d'Henry Bauchau, née d'un mal-être personnel et dans le sillage d'une analyse, constamment sous-tendue par le souci de traduire l'expérience du désastre autant que l'espérance de sa traversée en quête d'«un plus de vie dans un plus de sens» ${ }^{\mathbf{3 6 4}}$, se construit sur un partage émotionnel investi de la conviction

362 Texte du dossier de presse de Diotime et les lions, création au Théâtre des Osses, Fribourg, 1994 [D233]

363 Dominique Maingueneau, Le Discours littéraire. Paratopie et scène d'énonciation, Paris, Armand Colin, 2004.

364 Henry Bauchau, L'Écriture et la circonstance, Louvain-la-Neuve, Faculté de Philosophie et Lettres, 
profonde que ce partage, déjà, surmonte la douleur. Sur cette foi repose l'acte poétique, le pari de son geste autant que la possibilité même de son efficacité. Ce que Gisèle Sallin nomme "l'acte» théâtral tient tout entier à un investissement de confiance dans les possibilités de la parole ou du chant à agir concrètement, à affecter le locuteur parce qu'il s'auto-affecte en les produisant, et à toucher l'auditeur qui est atteint, et par là transformé, voire éveillé. Les comédiens eux-mêmes lui témoignent par ailleurs, pour ce qui concerne leur expérience propre, que l'interprétation des textes de Bauchau a été, pour eux, le lieu d'une modification sans retour de leur rapport au texte, à la langue et à eux-mêmes et, par là, d'une transformation de leur compréhension de leur métier ${ }^{\mathbf{3 6 5}}$.

La langue de Bauchau est émotionnellement chargée, et c'est en cela qu'elle émeut, qu'elle peut mouvoir, mettre en mouvement. Or, cette langue est le processus d'une longue décantation poétique, Henry Bauchau n'arrivant au degré de limpidité de son écriture qu'au fil d'un patient travail d'émondage, de simplification, de réduction à l'essence. C'est ce que ressent Gisèle Sallin lorsqu'elle décide de porter le texte à la scène en le gardant intégralement, sans y changer ni y retirer un seul mot, car le fragile équilibre de la parole juste, de ce qu'elle appelle le «juste chant", y serait perdu.

La haute exigence de ce travail est ce qui distingue le créateur, homme de folie en ce qu'il s'abstrait de la satisfaction ordinaire, pour prendre le rôle solitaire de vigie ou d'éveilleur, qu'il soit le rédacteur de l'œuvre ou celui qui la porte à la scène, ce qui en définitive leur assure une forme de complicité. "Sachez", écrit Gisèle Sallin à Henry Bauchau, "que désormais je veille sur vous, par vous et pour vous, je suis en état de veille et vous pouvez poursuivre votre folie» (01/10/1994, [B5318]). La correspondance entre l'écrivain et le metteur en scène témoigne en ce sens d'un échange d'expériences similaires : là où le romancier évoque la solitude du travail à la table d'écriture comme condition d'une concentration nécessaire à la création ("Je mène pour le moment une vie solitaire favorable au travail qui est la seule issue", 26/05/1997 [B5409]), Gisèle Sallin pour sa part, compare sa situation en période de mise en scène à celle d'une séquestration au "couvent" (Depuis quelques années, j'ai accepté, durant ces périodes de mises en scène, de n’avoir plus ni mari, ni femme, ni enfants, ni mère, ni amis, ni amours. [...] Durant six à huit semaines, je suis le dépositaire solitaire de tous les spectateurs à venir", 21/11/1996, [B5401]).

Concrètement, les échanges prennent aussi la forme d'un retour sur le travail de l'un et de l'autre: Gisèle Sallin envoie à Henry Bauchau ses impressions de lecture de ses livres [B5378, B5417, B5425], et réciproquement, le romancier qui assiste aux spectacles témoigne de ce que voir ses œuvres "sur la scène, par vos yeux" éclaire pour lui [B5378]. Au cœur de ce dialogue, on comprend que ce qui

1988, "Chaire de poétique», p. 77, repris dans L'Écriture à l'écoute, Arles, Actes Sud, 2000, p. 95. 365 Entretien avec Gisèle Sallin, 9 février 2010. 
lie profondément leurs démarches est que l'un comme l'autre visent dans l'art, non une évasion dans l'ailleurs mais une pleine appropriation du réel, et ne s'intéressent, pour reprendre les termes de Gisèle Sallin, "pas à l'impossible, mais à tous les possibles» [B5425] qu'ouvre la création.

\section{Diotime et Clios : le sens du geste juste}

Le Théâtre des Osses mettra en scène deux textes narratifs d'Henry Bauchau: Diotime et les lions en 1994, récit publié isolément du roman Edipe sur la route, qui sera joué une centaine de fois en Suisse, en Belgique, en France et au Québec; Clios le bandit, qui correspond au chapitre "Alcyon" du même roman, et qui, pour des raisons scénographiques sur lesquelles nouse reviendrons, ne sera représenté qu'à Givisiez en novembre et décembre $2008^{\mathbf{3 6 6}}$. Deux récits en «je» qui sont des anamnèses, c'est-à-dire la relation rétrospective de l'évolution du personnage: Diotime explique, dans son âge mûr, comment elle est passée de l'adolescence sauvage et exigeante à la maturité apaisée, et Clios, devenu bandit des grands chemins, violeur et pilleur, relate comment il a perdu son innocence de jeune berger pour devenir un guerrier sanguinaire, puis un hors-la-loi sauvage et cruel. Ces deux monologues se présentent donc en chiasme sur le plan des valeurs, mais en parallèle sur le plan de la dynamique transformationnelle qui les sous-tend: un être revient sur son passé, et plus précisément sur les années déterminantes de sa jeunesse, pour témoigner de la radicale transformation de son être qui a subi un déracinement et en a souffert, et pour dire comment il y a tout gagné, ou tout perdu, en termes de bonheur.

Ces deux textes jouent pleinement sur l'auto-affection du sujet parlant : le hérosnarrateur est amené à repasser par les phases d'exaltation et de détresse qui ont déterminé son parcours de vie, son initiation par la traversée d'un espace de mort dont il est ressorti grandi. Ce sont par ailleurs deux histoires passionnelles contrariées. On y trouve les plus belles pages d'histoire d'amour dans l'œuvre de Bauchau, la sensualité y affleure comme rarement dans cet univers où le bonheur charnel reste généralement dans l'écrin secret du non-dit. Les textes jouent sur le détail allusif, sur l'évocation suggestive et non démonstrative, d'où leur puissance dramatique à la scène: un mot résonne de tout un pan d'imaginaire laissé ouvert à la potentialité fantasmatique des auditeurs. Gisèle Sallin ne s'y est pas trompée, qui a refusé de tailler dans le récit et a imposé aux comédiens, Véronique Mermoud et Olivier Havran, l'intégralité du texte: dans ces deux ensembles, aucun mot n'est superfétatoire et le récit repose sur l'équilibre des résonances ménagées entre des expressions pesées, des termes comptés. Bauchau, qui pratique par ailleurs l'art de

366 À quoi il faut ajouter un cabaret-théâtre sur le thème des frères ennemis dans l'œuvre de Bauchau, mais qui n'a pas l'ampleur des deux mises en scènes commentées ici. 
la poésie hermétique, a ici ciselé deux textes où chaque mot pèse et soutient une structure événementielle et émotionnelle savamment agencée, et qui progresse inéluctablement vers un dénouement de retour à une forme d'équilibre qui ne nie pas la violence, mais la contient. Les derniers mots de Diotime sont: "Ce lieu, avec les rochers et l'espace brûlant qui l'entourent, est demeuré sacré. Si des lions et des hommes s'y rencontrent, aucun n'attaque et nul ne fuit. „ ${ }^{\mathbf{3 6 7}}$ Et Gisèle Sallin fait se clôturer Clios le bandit sur le dernier mot du monologue du héros: "Jusqu'au moment où je vous ai rencontrés, j'ai vécu de séduction, de vols, de pillages, nourrissant ma haine et ma honte de crimes toujours plus inutiles " ${ }^{\mathbf{3 6 8}}$; le texte de Bauchau se poursuit dans le roman par: "Il y a un silence, la mer déferle sur les récifs et vient battre la falaise. Du côté de la campagne, on entend le cri d'une chouette; Antigone pleure, la tête contre l'épaule d'CEdipe. „369 La mise en scène traduit ces éléments par le silence de Clios et l'arrivée d'une mince coulée d'eau, qui peut tout aussi bien figurer le jaillissement d'une source au milieu du paysage dévasté que celle des larmes de la terre... ou des auditeurs.

La mise en scène construit donc sa forme de fidélité au texte non seulement par la reprise sans retouche du texte de l'écrivain, mais aussi par les trouvailles de sa mise en espace par le scénographe Jean-Claude de Bemels. Dans Diotime et les lions, le décor repose entièrement sur le jeu avec un lattis de bambous qui, relevé à la verticale, forme un mur et peut symboliser la rudesse d'un monde patriarcal auquel se heurte la jeune hérö̈ne, mais qui, disposé à l'horizontale, peut simuler la protection, ou encore se disposer en diagonales et en courbes, se faire souple, mouvant, mystérieux, et suggérer par des jeux d'ombre et de lumière une intimité féminine. "Le décor", écrit un journaliste, "d'une sobriété quasi spartiate mais d'une qualité esthétique rare, vit sur scène accompagnant et soutenant la narration " ${ }^{\mathbf{3 7 0}}$. "On a dit du décor qu'il se réduit à peu de chose», ajoute Éliane Waeber, "mais ce peu de chose est vivant: grand hamac en cylindre de bois qui mime la jungle ou la savane, devient lieu clos, couche, harpe. La musique est de la même veine: on doit à Max Jendly une rumeur légère, tantôt fluide, tantôt saccadée. Comme un écho amical de l'émotion de Diotime " ${ }^{371}$. Elle souligne "la minutie de la mise en scène, les prouesses du régisseur qui fait se déployer, se cabrer, respirer le décor, seul interlocuteur de Diotime „372, comme la musique composée sur mesure. Ces commentaires montrent à quel point le visible et l'audible, ici, sont accordés aux caractéristiques verbales de la langue de Bauchau. Quant à la comédienne Véronique Mermoud, elle doit porter le texte en trouvant une justesse de ton qui

367 Henry Bauchau, Diotime et les lions, Arles, Actes Sud, 1991, "Babel», p. 63.

368 Henry Bauchau, Edipe sur la route, Arles, Actes Sud, 1990, "Babel», p. 87.

369 Ibid., p. 88.

370 MJN, "Les planches du Petit La Faye vont brûler au feu de Diotime», dans La Liberté, 23 novembre 1994.

371 Éliane Waeber, "Véronique Mermoud interprète Diotime avec intensité et sans concession", dans La Liberté, 20 avril 1994.

372 Idem. 
puisse moduler son identité, tantôt de femme qui a atteint la sagesse, tantôt de gamine pleine de fougue, de dépit ou de rage; le Prix Sacha Pitoëff qu'elle remporte pour cette performance fait comprendre à quel point la comédienne a réussi ce pari difficile.

En droite ligne de ce que Gisèle Sallin dit avoir hérité de son apprentissage auprès de Maria Casarès, tout vise donc le "geste juste». Un critique de théâtre précise que chez Gisèle Sallin, "le matériau n'est jamais décoratif " ${ }^{373}$. Il en va en effet ainsi aussi dans Clios le bandit, au point de sacrifier à cette justesse la possibilité de déplacer le spectacle. Car le texte de Clios, cet écorché vif, qui s'est retrouvé enrôlé à son corps et à son coeur défendant dans une impitoyable guerre de clans, qui y a perdu ses biens, sa famille et jusqu'au sens de son identité, qui a sans le vouloir tué l'homme qu'il aimait le plus au monde dans le chaos d'un incendie guerrier, et dont les valeurs ont été bafouées, piétinées, trahies, vit désormais comme une bête traquée dans un cercle de violence et de haine qu'il ne cesse d'alimenter lui-même en s'y brûlant. Gisèle Sallin choisit de visualiser sa situation intérieure en plaçant le spectacle dans un décor de forêt calcinée, absolument intransportable. Si l'incendie de la forêt joue bien un rôle capital dans l'aventure de Clios, elle est aussi, rappelons-le, un topos privilégié de l'imaginaire d'Henry Bauchau, qui présente la scène d'incendie de la ville de Louvain lors de l'invasion allemande, vécue par lui dans sa première enfance sans rien y comprendre, comme un "événement bouleversant» et un "point essentiel» de son existence, où s'ancre sa création littéraire ${ }^{374}$.

Elle confie le rôle à un jeune comédien qui lui semble être celui que ce texte attendait pour pouvoir être monté à la scène: "Mon désir de monter Clios le bandit», dit-elle, "est né tout de suite après Diotime et les lions, mais il a fallu 15 ans pour que les circonstances soient réunies. L'une d'elles fut l'arrivée du comédien Olivier Havran au Théâtre des Osses. Pour prendre en charge un texte de ce niveau, cela nécessite de la part de l'interprète un solide travail personnel et beaucoup de temps. ${ }^{375}$

Revenons dès lors sur le long développement qui a permis à ce projet d'atteindre sa maturation. Edipe sur la route est, on le sait, le livre par lequel Gisèle Sallin est entrée dans l'univers littéraire d'Henry Bauchau. Le roman relate l'épisode laissé dans l'ombre par Sophocle entre CEdipe roi et CEdipe à Colone, et Clios fait partie des personnages inventés par le romancier contemporain pour comprendre le sens du cheminement du héros thébain banni jusqu'au moment de sa réhabilitation

373 "Désir d'être", dans Scènes magazine n77, mai 1994.

374 Henry Bauchau, L'Écriture et la circonstance, [1988], repris dans L'Écriture à l'écoute, op. cit., pp. 54-55. Voir aussi: "La ville brûle avec ses arbres, avec les feuilles de l'été, avec ses greniers et ses meubles. Elle fait feu, elle allume ton incendie avec les livres de la bibliothèque de l'université./ La ville a pris feu dans ta vie qui commence, elle s'effondre sur elle, avec ses milliers de poutres et de maisons", "La Sourde Oreille ou le Rêve de Freud " [1978], dans Poésie complète, Arles, Actes Sud, 2009, p. 230.

375 Gisèle Sallin, Gisèle Sallin et Henry Bauchau. op. cit. 
comme personnage sacré. Mais le bandit n'est jamais apparu à Gisèle Sallin comme une figure secondaire, tout au contraire, puisque sans lui, le livre de Bauchau lui est tombé des mains:

La lecture de ce livre fut pour moi un choc. Je ne voulais plus le lâcher. Mais lorsque le personnage de Clios a soudain quitté le roman, j’ai été vraiment fâchée contre l'auteur. J'ai d'ailleurs posé l'ouvrage pendant un temps, et je ne l'ai repris que lorsqu'une amie, qui avait terminé le livre, m’a dit que Clios revenait à la fin. ${ }^{376}$

Avant de se centrer sur le seul monologue de Clios, Gisèle Sallin a pensé mettre en scène l'intégralité du roman Edipe sur la route, qu'elle a imaginé, dès août 1994 [B5305 et s.], présenter comme une tétralogie. Elle se montre très convaincue ("ÇA AGIT à plusieurs niveaux", écrit-elle au romancier (fax du 03/06/1995, [B5340]. Aussi organise-t-elle une lecture probatoire en Suisse, en présence de l'auteur, le 8 juin 1995 [B5335]. Henry Bauchau marque son accord de principe, mais non sans réserve quant au déploiement d'énergie qui lui semble démesuré: il met en garde contre "le risque de vous faire entrer dans une tâche très lourde dont l'heureux aboutissement [est] peu sûr" (lettre du 24 janvier 1006, [B5378]). En juin 1996, Gisèle Sallin, qui a l'enthousiasme tenace, pense présenter la pièce, désormais intitulée Quand le roi aveugle entendit chanter sa couronne, au concours de la Fondation Onassis (lettre du 20/06/1996, [B5386]. Il n'y aura pas de suite à ce projet, mais la mise en scène de Clios le bandit apparaît, tenant compte de cet historique, comme le résultat d'une décantation de quinze années, ce qui ne manque pas de souligner son importance symbolique dans le parcours du Théâtre des Osses, qui créera finalement ce spectacle à l'occasion de ses trente ans d'existence et de ses vingt années d'implantation à Givisiez.

Sa mise en scène se présente à cet égard comme l'aboutissement d'un processus de réflexion de longue haleine quant à la théâtralité du texte de Bauchau. Le récit de Clios s'adresse à CEdipe, l'ancien roi de Thèbes qui a empêché le bandit de violer Antigone et qui, sur l'injonction suppliante de celle-ci, a renoncé à le tuer. Clios, décontenancé par ce geste inattendu qui vient rompre le cercle infernal de la violence, s'attache au roi aveugle et entreprend de lui raconter son histoire, qui est celle de sa déchéance. C'est aussi celle de sa traversée de la mort et de sa renaissance: une initiation. On est dans la langue du pâtir, du pathos, celle de la recherche d'une écoute, de la possibilité d'être reçu, de redevenir recevable. Le roman ne montre aucune réaction d'Edipe, mais on voit qu'il donne à tout le moins au tueur la possibilité de s'exprimer et à Antigone de se répandre en larmes. Edipe devient ainsi, pour ses deux compagnons de route, le déliant.

Or c'est exactement ainsi que Gisèle Sallin et Jean Claude de Bemmels décident de positionner le public tout autour de Clios, comme si un feu de camp réunissait les spectateurs autour de lui pour l'écouter en compagnie d'CEdipe, ou même à sa

376 Idem. 
place. Ce dispositif fait entrer dans l'espace calciné de la vie du héros; il induit à éprouver que chacun peut, comme le vieil aveugle, à la faveur d'une halte imprévisible comme celle qu'a provoquée Antigone, prendre la mesure de l'importance d'arrêter le cercle vicieux de la violence et de se mettre en position d'écouter, de recevoir. Alors une source rejaillit, ou des larmes, parce qu'un mouvement reprend. La scénographie du texte s'accorde ici exactement à l'acte qu'il figure. Car ce n'est pas seulement dans le décor qu'elle nous convie à pénétrer, mais dans l'acte. Un acte qui peut avoir sur le spectateur une force d'éveil.

On comprend dès lors la rencontre naturelle de Gisèle Sallin avec l'œuvre d'Henry Bauchau dont toute la dynamique interne est celle de l'initiation. On saisit ce qui rassemble ces créateurs: à son tour, l'action du Théâtre des Osses, en s'accordant intimement, mais aussi publiquement, à de tels textes, initie, selon les termes par lesquels sa directrice traduit sa propre initiation au théâtre:

Elle m'a transmis dans l'espace magnifique du théâtre des secrets: ... l'ÉVEIL.

L'éveil à la force... à la puissance du geste juste

L'éveil à la grâce du chant intérieur. [B5272 bis]

En définitive, si l'œuvre d'Henry Bauchau a porté plus loin le Théâtre des Osses, le Théâtre des Osses a porté plus loin l'œuvre d'Henry Bauchau. On pense à cet échange entre Antigone et Clios, qui dit: "Je suis heureux de t'avoir rencontrée. J'irai plus loin, grâce à toi. „377

Myriam Watthee-Delmotte

FNRS/Université catholique de Louvain

377 Henry Bauchau, Antigone, Arles, Actes Sud, 1997, "Babel», p. 157. 